Conclusion To diagnose the condition history is crucial, while laboratory and gastroscopy add more strength. Fluticasone inhaler, exclusion diets and montelukast help in the majority of patients. Strictures associated with EoE can be successfully treated with biodegradable stent.

Competing interests None declared.

\section{PWE-181 PANCREATIC EXOCRINE FUNCTION AFTER MAJOR UPPER GASTROINTESTINAL SURGERY MEASURED WITH A CARBON 13 MIXED TRIGLYCERIDE BREATH TEST}

doi:10.1136/gutjnl-2012-302514d.181

${ }^{1,2} \mathrm{~S}$ Ward, * ${ }^{1} \mathrm{R}$ Cade, ${ }^{1,2} \mathrm{~S}$ Mackay, ${ }^{1} \mathrm{~S}$ Hassen, 'S Banting, ${ }^{2} \mathrm{P}$ Gibson. ${ }^{1}$ Upper Gl Surgery, Eastern Health, Melbourne, Australia; ${ }^{2}$ Monash University, Melbourne, Australia

Introduction Major upper gastrointestinal resectional surgery, including oesophagectomy, gastrectomy and pancreatico-duodenectomy, can result in post-operative nutritional difficulties, which may in part be associated with reduced pancreatic exocrine function, but there is debate in the literature about the actual proportion of these patients that have pancreatic exocrine insufficiency which may then benefit from oral supplementation. The Carbon 13 labelled mixed triglyceride breath test (C13-MTG-BT) was used to indirectly measure pancreatic exocrine function in post-operative patients and these results were compared to the test performed in control subjects.

Methods 30 normal subjects, 15 post-oesophagectomy patients, 15 post-gastrectomy patients and 10 post-pancreatico-duodenectomy patients were recruited to undertake the C13-MTG-BT at Box Hill Hospital, Melbourne, Australia, between August 2009 and January 2011. The C13-MTG-BT was performed using $200 \mathrm{mg}$ of C13-MTG substrate and measured using an infra-red isotope spectrometer. The cumulative percentage of ingested $\mathrm{C} 13$ exhaled after $6 \mathrm{~h}$ and the time at peak rate of C13 excretion were measured in all subjects and compared between groups, with statistical significance calculated using the Student $t$ test.

Results The mean cumulative percentage of ingested C13 exhaled after $6 \mathrm{~h}$ in the control group was $28.6 \%$ with a SD of $8.8 \%$. The cumulative percentage exhaled after $6 \mathrm{~h}$ in each post-operative group compared with the control group was not significantly different. The time of peak rate of C13 excretion was earlier in the post-operative groups compared with the control group.

Conclusion This study has not found a large percentage of patients post major upper gastrointestinal resections with measurable reduction in pancreatic exocrine function using the C13-MTG-BT, which is in contrast to the literature. The finding of earlier times of peak rate of excretion imply the post-operative patients tend to have more rapid gut transit. Larger and prospective studies using this test or another pancreatic function test may be useful to detect the proportion of post-operative patients with pancreatic exocrine insufficiency that may then benefit from pancreatic exocrine supplementation.

Competing interests None declared.

\section{PWE-182 EFFICACY OF ARGON PLASMA COAGULATION THERAPY FOR GASTRIC ANTRAL VASCULAR ECTASIA}

doi:10.1136/gutjnl-2012-302514d.182

S Salunke, * P Phull. Gastrointestinal \& Liver Service, ABERDEEN ROYAL INFIRMARY, Aberdeen, UK

Introduction Argon plasma coagulation (APC) is an established endoscopic therapy for various gastrointestinal disorders but with varied success in the management of Gastric Antral Vascular Ectasia (GAVE). The aim of this study was to assess the efficacy of APC in the management of patients with GAVE.

Methods Retrospective audit of upper GI endoscopies (UGIE) performed at our institution between 1 January 2007 and 31 December 2011. Data were extracted from the endoscopy reporting software (Unisoft) database using search term "argon plasma coagulation." The case notes were reviewed for those patients undergoing APC for a diagnosis of GAVE.

Results 306 episodes of APC were noted on 127 patients. Of these 135 APC sessions were performed on 30 patients for management of GAVE. Of the 30 patients 17 (57\%) were women. The median age was 68 (range 44-89) years. Co-morbidities included chronic kidney disease in $9(30 \%)$, chronic liver disease in $7(23 \%)$, malignancy in 4 $(13 \%)$, scleroderma in $3(10 \%)$. Seven $(23 \%)$ patients were on Aspirin, 1 (3\%) on Clopidogrel, 1 (3\%) on Clopidogrel and Aspirin and $2(7 \%)$ on Warfarin. The indication for index UGIE was anaemia in $23(77 \%)$ cases, iron deficiency without anaemia in $2(7 \%)$ and acute upper gastrointestinal bleeding in $5(17 \%)$. APC therapy was applied at 50-65 watts. A cluster of multiple sessions of APC was treated as "one cycle" of therapy. APC was applied with success in single cycle lasting over a median period of 2 (range $0-30$ ) months with median of 3 (range 1-24) APC sessions. Six (20\%) patients required a single session of APC. The median follow-up period was 26.5 (range $1-59)$ months. We noted mean haemoglobin $(\mathrm{Hb})$ rise of $22 \%$ and $\mathrm{Hb}$ normalised in $57 \%$ cases. In $3(10 \%)$ patients symptomatic anaemia recurred and two of them required one further cycle and one required two cycles of APC therapy. There was no significant difference in the index $\mathrm{Hb}$ and number of APC sessions required between men and women. However, 13 out of 17 (75\%) women normalised their $\mathrm{Hb}$ at the end of therapy while only four out of $13(31 \%)$ of men could achieve normal $\mathrm{Hb}$ at the end of treatment.

Conclusion Argon plasma coagualtion appears to be an effective therapy for patients with Gastric Antral Vascular Ectasia.

Competing interests None declared.

\section{PWE-183 INVESTIGATIONS FOR COELIAC DISEASE IN IRON DEFICIENCY ANAEMIA-ARE WE FOLLOWING BSG GUIDELINES?}

doi:10.1136/gutjnl-2012-302514d.183

${ }^{1} \mathrm{~S}$ Nayagam, ${ }^{2} \mathrm{~K}$ Lloyd, ${ }^{2} \mathrm{E}$ Byrne, ${ }^{2} \mathrm{M}$ M Walker, ${ }^{1} \mathrm{H}$ R T Williams. ${ }^{1}$ Department of Gastroenterology, St Mary's Hospital, Imperial College NHS Trust, London, UK; ${ }^{2}$ Centre for Pathology, Imperial College, London, UK

Introduction BSG guidelines for the investigation of iron deficiency anaemia $(2011)^{1}$ recommend screening of these patients for coeliac disease (CD) by serology. Small bowel biopsy is recommended at OGD if coeliac serology is positive or not available. If coeliac serology is negative, small bowel biopsies need not be performed at OGD unless there is a high degree of suspicion for CD despite negative serology.

Methods We retrospectively evaluated the use of coeliac serology testing (tissue transglutaminase antibody, tTG) and upper gastrointestinal endoscopy (OGD) with duodenal biopsies in the evaluation of anaemia, according to BSG guidelines in our NW London teaching hospital cohort. All upper GI endoscopies performed for anaemia over a 6-month period were reviewed for rates of duodenal biopsy and results, and serological testing.

Results In 6 months, 206 OGDs were performed for anaemia. Duodenal biopsies were taken in 134/206 (65\%). Of $72(35 \%)$ procedures at which no duodenal biopsy was taken, another cause for anaemia found on OGD in 27, six had melaena or acutely falling haemoglobin as an indication for OGD, six had previous duodenal 